\title{
MATERIALS THAT IMPROVE THE SHIELDING EFFICIENCY FROM EM RADIATION
}

\author{
Stanko Colak, Damir Varevac \& Ivana Milicevic
}
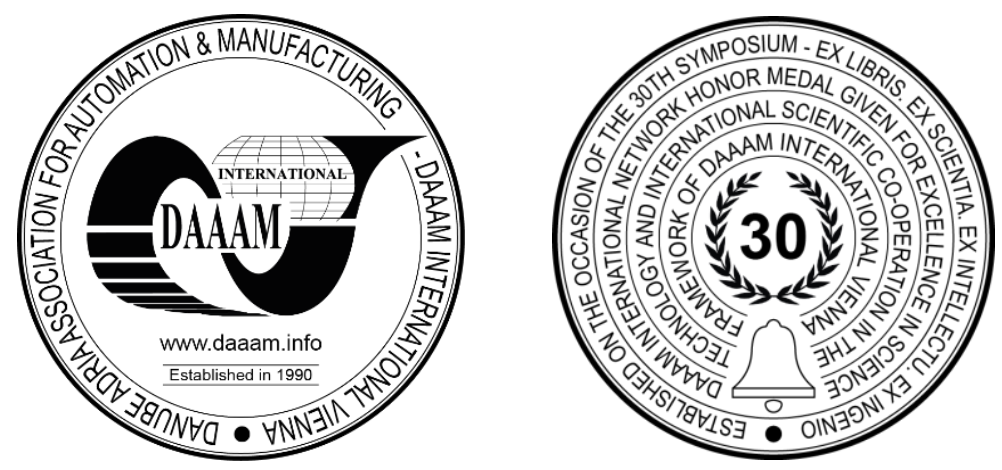

This Publication has to be referred as: Colak, S[tanko]; Varevac, D[amir] \& Milicevic, I[vana] (2020). Materials that Improve the Shielding Efficiency from EM Radiation, Proceedings of the 31st DAAAM International Symposium, pp.0800-0806, B. Katalinic (Ed.), Published by DAAAM International, ISBN 978-3-902734-29-7, ISSN 1726-9679, Vienna, Austria

DOI: $10.2507 / 31$ st.daaam.proceedings. 111

\begin{abstract}
The field of research of this paper are materials used to improve the properties of building materials on the penetration of EM radiation. The danger of EM radiation is encountered in everyday life, especially in today's time when technology is advancing more and more and over time the danger of radiation is increasing. The improvement of material properties at EM radiation penetration is best described by the shielding efficiency (SE), which is defined as the sum of the loss of absorption during wave transport in the medium and the loss of reflection for waves inside and outside the shielding material. The following materials were treated: metallurgical slags, steel mini scale (ms) waste, cement paste, fly ash, carbon nanotubes and composite materials for protection against electromagnetic interference.
\end{abstract}

Keywords: shielding efficiency (SE); steel mini scale (ms) waste; metallurgical slags; fly ash and carbon nanotubes.

\section{Introduction}

As technology evolves and the danger of EM radiation increases, it is increasingly necessary to use materials that have the ability to absorb EM radiation. This paper describes some materials used to improve the properties and protection of building materials against the penetration of electromagnetic (EM) radiation. Since the project for the innovation of these materials is only in its infancy, the paper describes the materials that have been researched so far as well as their shielding efficiency according to various tests. In the second part, something general is said about the shielding efficiency (SE) materials against the penetration of electromagnetic radiation. The types of electromagnetic wave loss are described and the definition of the shielding efficiency of material on the penetration of electromagnetic radiation is explained.

The third part describes the influence of metallurgical slags on the protection of materials from electromagnetic radiation. Professor Costas Sikalidis has addressed the shielding efficiency of metallurgical slag in his research [1]. The two most famous metallurgical slags used to protect materials from electromagnetic radiation are described and these two slags are compared with each other. The influence of scrap tires as well as the influence of the mixture of the two most famous metallurgical slags on the increase of the efficiency of protection of materials from the action of electromagnetic radiation is also shown. 
In the fourth part, some materials reinforced with steel mini scale (ms) waste are described and compared. Part of the examination of Professor Costas Sikalidis was taken as an example. The fifth part describes composite materials for protection against electromagnetic radiation and shielding efficiency of cement with carbon. The sixth part describes the shielding efficiency of the material with fly ash. Electromagnetic radiation protection values were also compared for 4 samples of which three samples contained three fractions of fly ash while one sample contained a control cement paste. The seventh part describes the main types of carbon composites used for the purpose of electromagnetic shielding, namely carbon nanotubes and graphene. The types of carbon nanotubes are described and compared, as well as the influence of the thickness of the whole sample on the efficiency of protection of the whole sample reinforced with carbon nanotubes. A conclusion was written at the end of the paper.

\section{General information on the shielding efficiency of material on the penetration of EM radiation}

With the increasing use of electronic devices, people are concerned about the harmful health consequences that could be caused by all-day exposure to the electromagnetic fields of mobile telephone stations or radio and television transmitters [1]. The improvement in the material properties of EM radiation is best described by the shielding efficiency (SE). It can be concluded that different materials contribute to radiation protection in different ways. Protection from electromagnetic wave is a scheme that prevents the radiation of electromagnetic waves to a specific area or space. There are generally two types of electromagnetic wave loss:

- the loss in reflection of waves on the media material,

- the loss in wave transportation [2].

The loss in wave transport in the media material is also called the absorption loss. The total loss of electromagnetic waves during transport through the protective material is presented as the shielding efficiency (SE), and is obtained by summing the loss of reflection and loss of absorption:

$$
\mathrm{SE}=\mathrm{R}+\mathrm{A}
$$

where A represents the loss of absorption during the transport of waves in the medium, and R represents the loss of reflection which may include both reflections when the waves are inside and outside the shielding material [2]. Protection against electromagnetic interference (EMI) means the reflection and / or absorption of electromagnetic radiation by a material, which thus acts as a shield against the penetration of radiation through the shield.

\section{Metallurgical slags}

Metallurgical slags are used to protect clay-based ceramic building materials. The shielding efficiency of metallurgical slag examined by Professor Costas Sikalidis is briefly described here. There are two most common types of metallurgical slags: slag from an electric arc furnace slag (EAFS) and ladle furnace slag (LFS). Figure 1 shows that an electric arc furnace slag (EAFS) shows better material protection efficiency than the addition of ladle furnace slag (LFS).

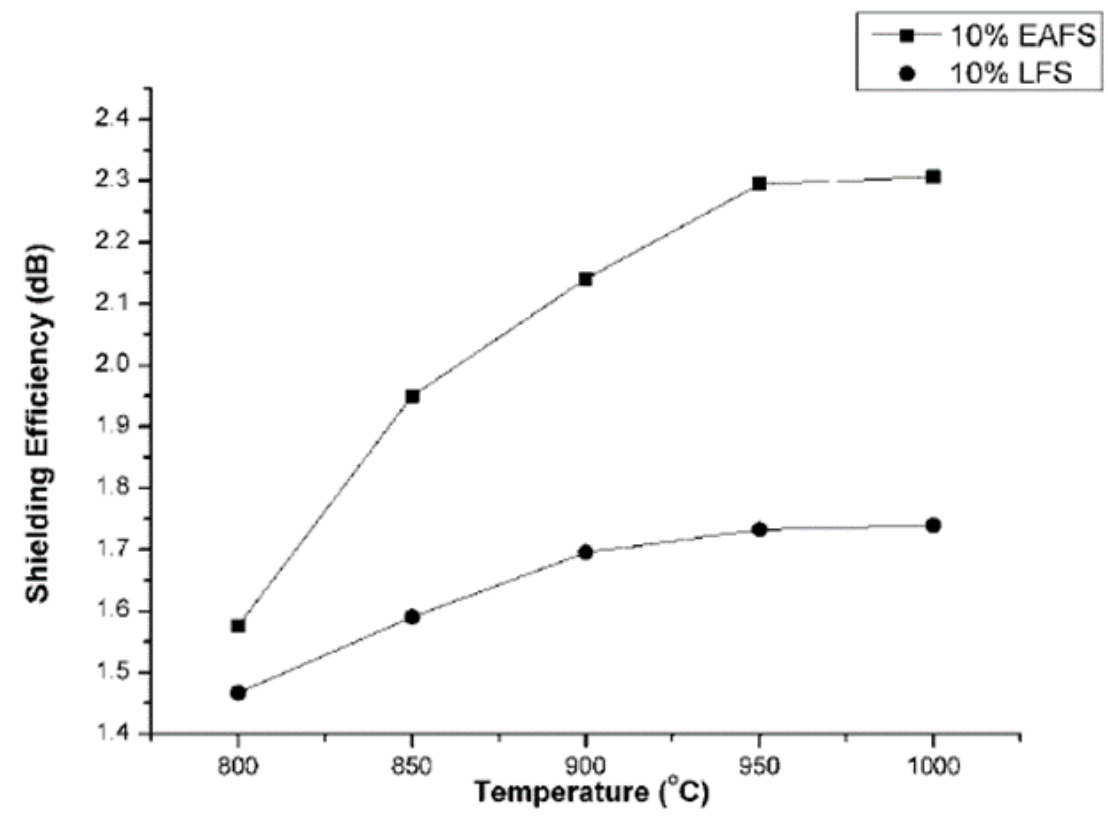

Fig. 1. Comparison of the impact of EAFS and LFS on the shielding efficiency (SE) of materials [3] 
Tire rubber wastes improve the shielding efficiency and the absorption and reflection coefficients of cement paste samples. In tests of mixtures of EAFS and LFS, it has been proven that EAFS, in addition to better shielding efficiency, has better absorption and reflection than LFS. It can be seen from Figure 2a that SE increased with increasing addition of tire rubber waste. Figure $2 \mathrm{~b}$ also compares three types of samples containing metallurgical slag: mixture 1 containing $25 \%$ EAFS and $75 \%$ LFS, mixture 2 containing 50\% EAFS and 50\% LFS and mixture 3 containing $75 \%$ EAFS and $25 \%$ LFS of the total sample weight. When it comes to the average shielding efficiency for these samples, we can see that the shielding efficiency is higher when the proportion of EAFS slag bigger is. LFS slag also contributes to shielding efficiency, and EAFS slag still has better shielding efficiency. As shown in Figure 2c, the absorption coefficient is increased by the addition of tire rubber waste. It can be seen from Figure $2 d$ that EAFS slag has a better effect on the absorption coefficient than LFS slag. Deviations of the reflection coefficient of cement pastes containing waste tires at $\mathrm{X}$-band frequencies are given in Figure 2e. The reflection coefficient is slightly increased by the addition of tire rubber wastes, although the general trend has remained the same. Figure $2 \mathrm{f}$ shows that EAFS increases the reflection of the material on the penetration of EM radiation more than LFS slag [4].

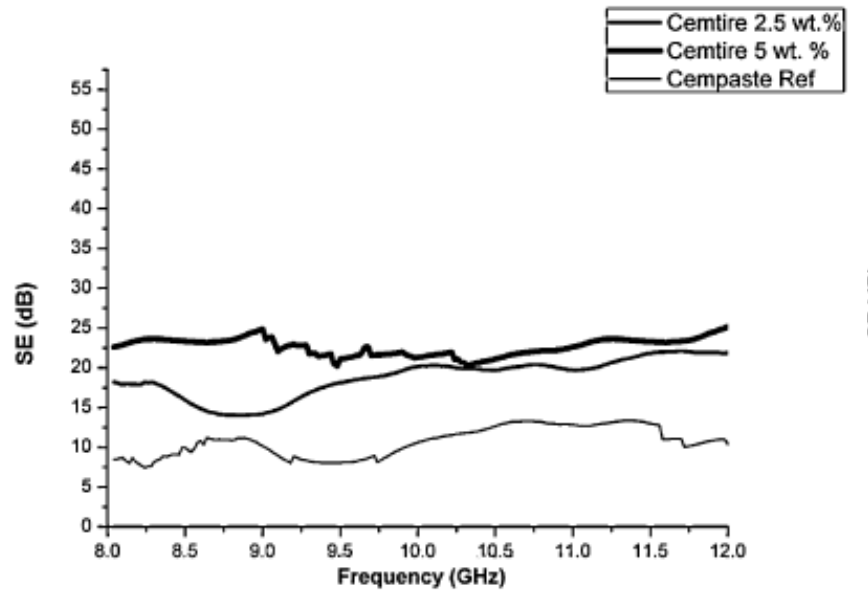

(a)

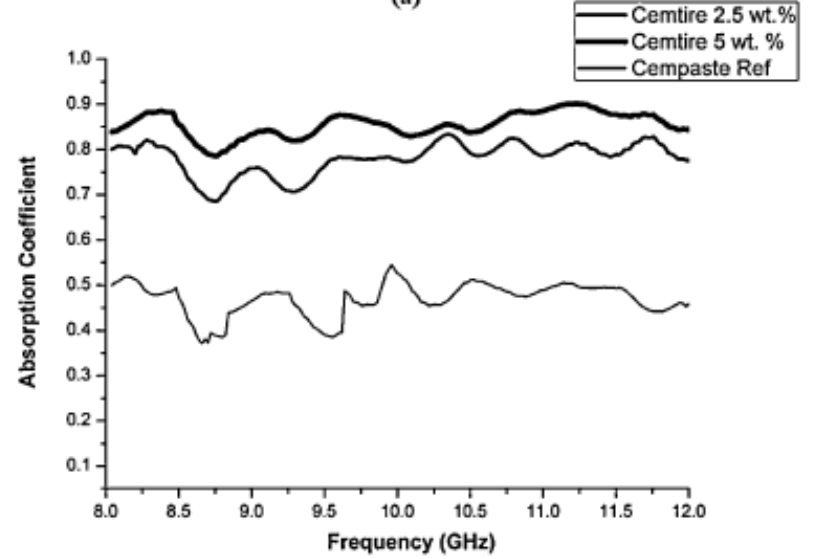

(c)

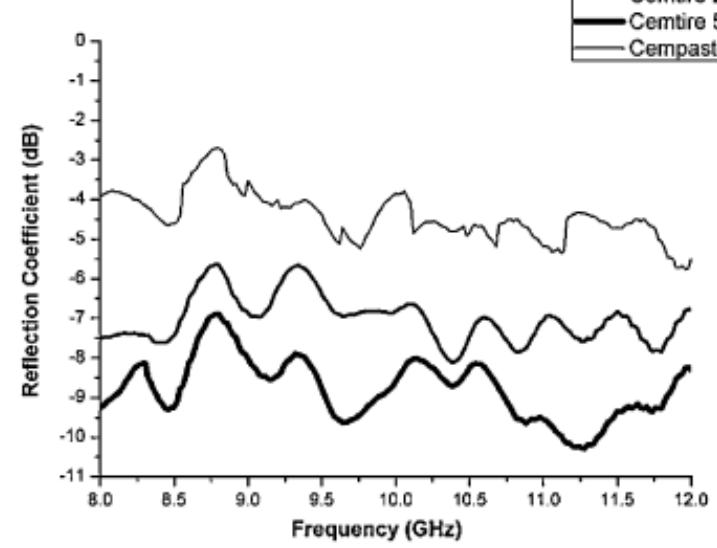

(e)

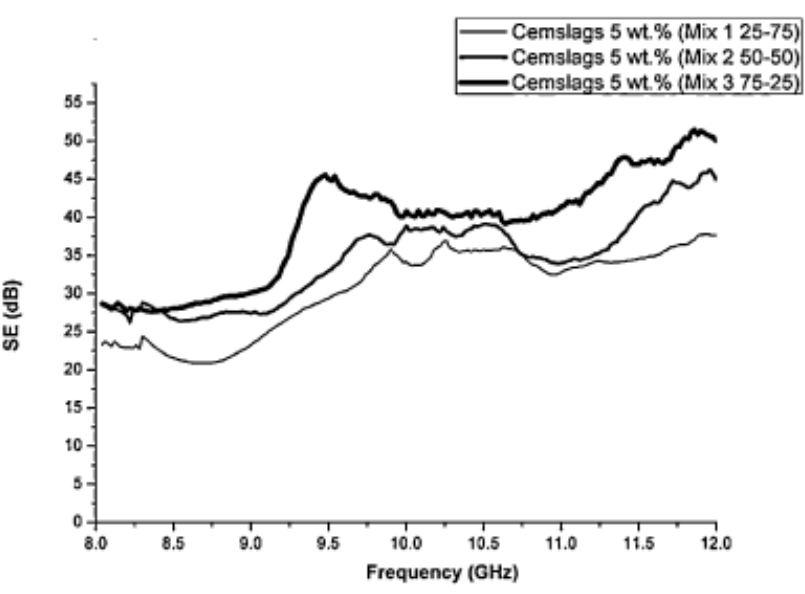

(b)

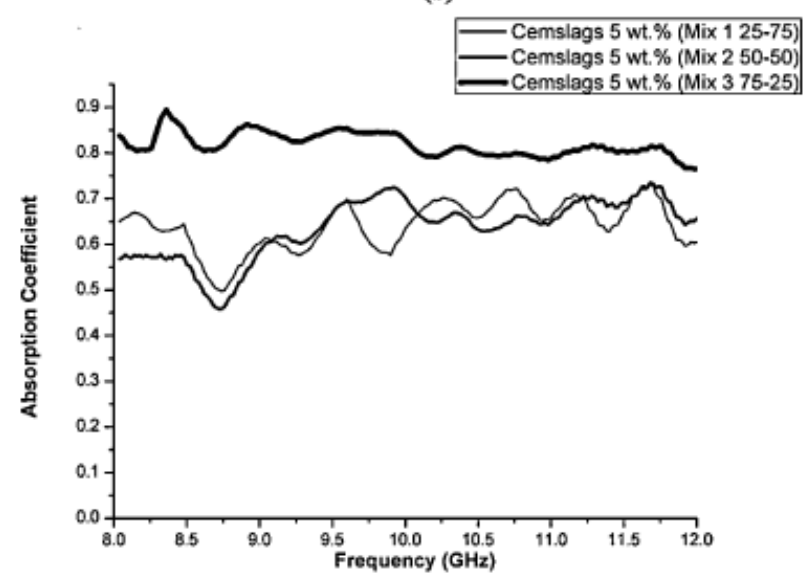

(d)

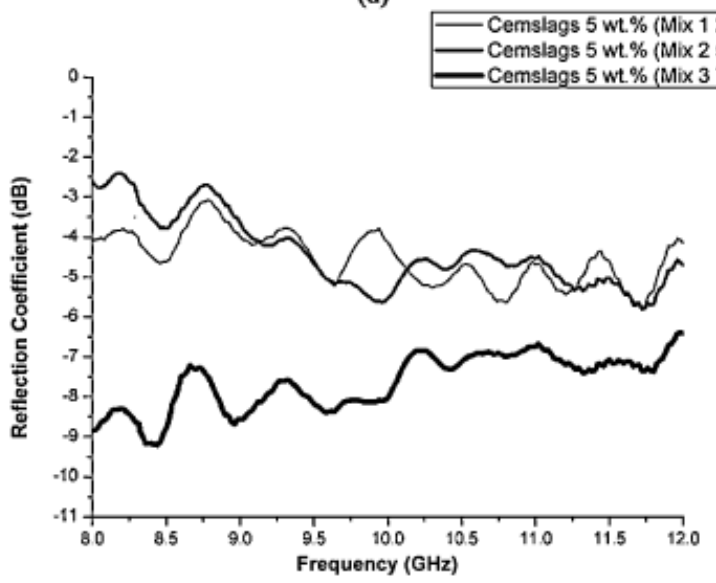

(I)

Fig. 2. (a-f) SE, variation of absorption and reflection coefficient of small cement paste walls containing mixed slags and tire wastes respectively at $8-12 \mathrm{GHz}$ frequency range [4] 


\section{Materials reinforced by the steel mini scale (ms) waste}

Professor Costas Sikalidis and his colleagues compared two samples that use steel mini scale (ms) waste as an additive. The first pattern is a brick wall pattern, and the second pattern is a pattern of two ceramic tiles stacked into a sandwich construction. Ceramic tiles have a better protective effect than a brick wall.

When it comes to absorption, a brick wall has a higher initial absorption coefficient than a ceramic wall, but with increasing frequency the absorption coefficient of a brick wall retains a similar value, while with ceramic tiles the absorption coefficient generally increases with increasing frequency, so at slightly higher frequencies of the ceramic tiles reinforced with steel scrap have on average a higher coefficient of absorption than a brick wall reinforced with steel scrap $[5]$.
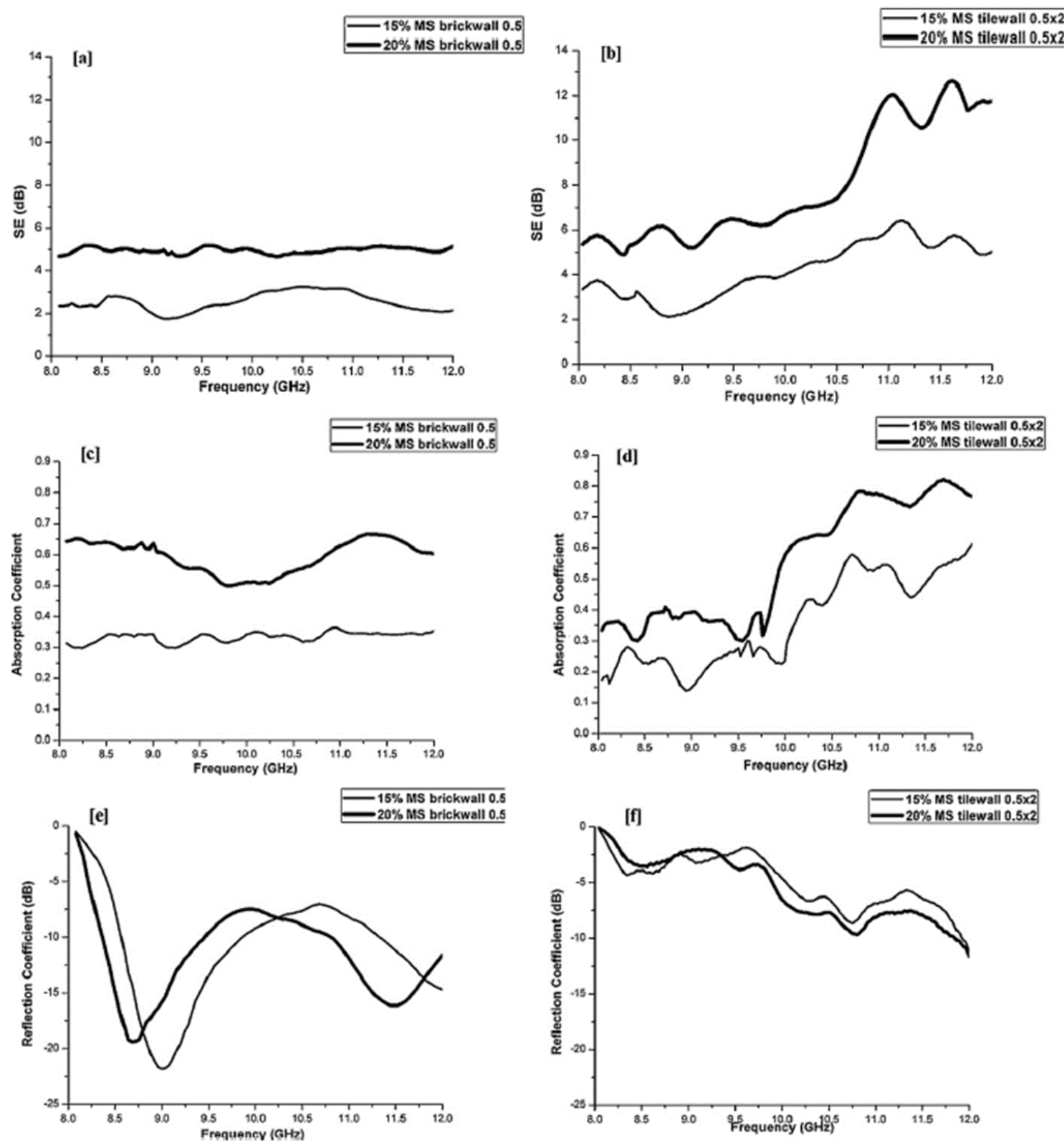

Fig. 3. Comparison of shielding efficiency (SE), absorption and reflection coefficient of brick walls and sandwich ceramic tile walls [5] 


\section{Composite materials}

The shielding efficiency of cement and its absorption property are very low. The addition of carbon materials will not improve the cement strength, but will greatly improve its electromagnetic properties. The sample of cement with the addition of carbon materials thicker is, that is the higher shielding efficiency of protection against EM interference. Although there are deviations from this rule, for example, in the examination of Lee et al. (2010), reinforced cement samples with a thickness of $4 \mathrm{~mm}$ have lower protection efficiency than samples with a thickness of $3 \mathrm{~mm}$. Figure 4 shows a comparison of cement samples of different thicknesses with different water-cement ratio.

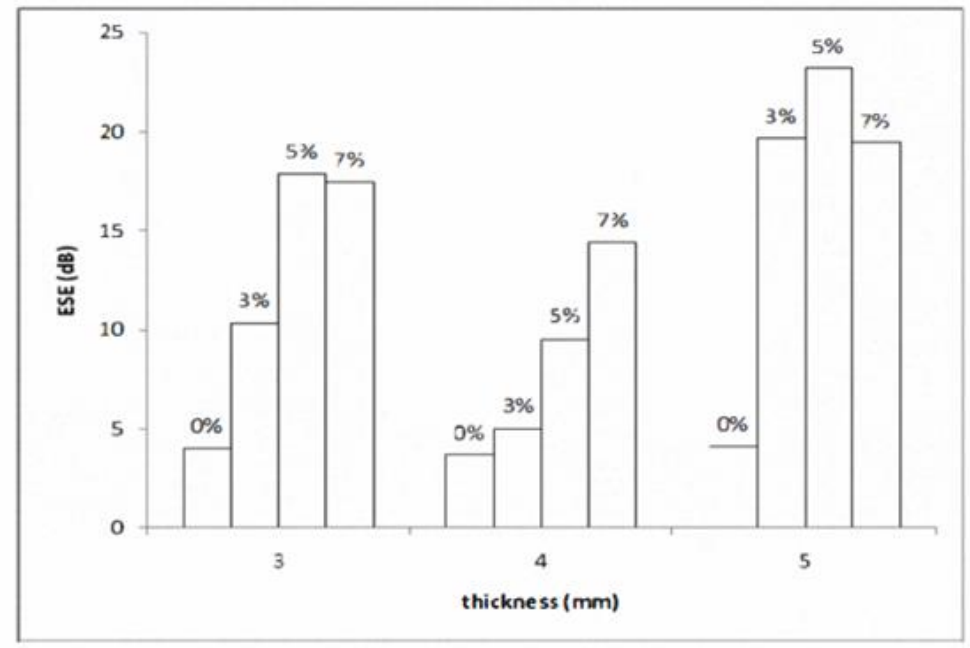

Fig. 4. Maximum values of shielding efficiency for samples of different thicknesses [2]

\section{Fly ash}

Fly ash brick consists of $15 \%$ fly ash and $85 \%$ clay. They are stronger and lighter than clay bricks. This type of brick is more useful than the usual clay bricks and fly ash. The use of fly ash as a mixture leads to an increase in the shielding efficiency against electromagnetic interference (EMI) from 4 to $8 \mathrm{~dB}$ at a frequency of $1 \mathrm{GHz}$. Adding a mixture of fly ash to the clay blocks reduces the swelling percentage and the swelling pressure. Atterberg boundary studies have shown that the addition of fly ash reduces the plasticity index by decreasing the liquid limit and increasing the plastic limit. The addition of the fly ash mixture improved the stabilized lime, reducing the adverse effects, the percentage and pressure of swelling, and the plasticity index.

Figure 5 shows the electromagnetic radiation protection values for 4 samples $4 \mathrm{~cm}$ thick of which three samples contain three fractions of fly ash while one sample contains a control cement paste. This study was performed by Zornoza et al. (2010) [7]. The figure shows that the effectiveness of protection increases with increasing frequency of radiation. Also, it is obvious that the original fly ash (FA0) gives the samples higher protection efficiency than the cement paste (Control paste). Non-magnetic fly ash (NMFA) gives samples lower protection efficiency than cement paste, while magnetic fly ash (MFA) gives higher protection efficiency than original fly ash and cement paste.

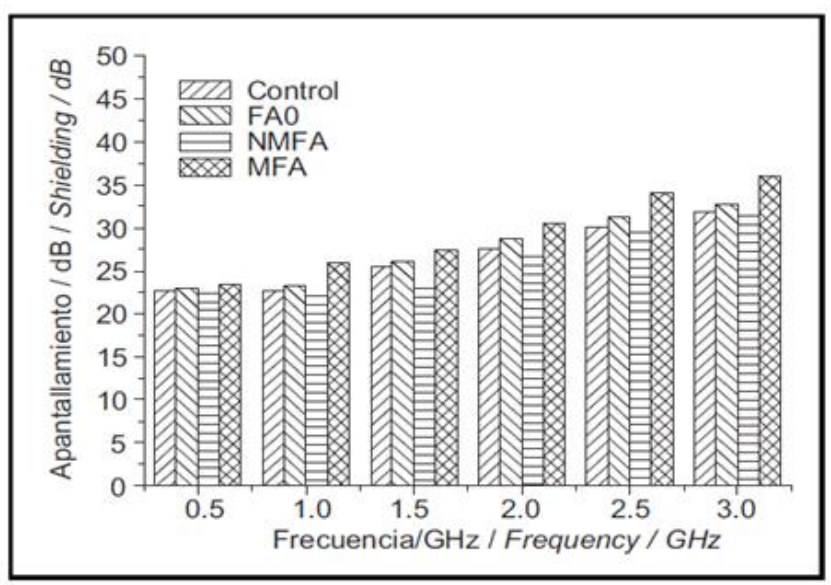

Fig. 5. EMI protection in $4 \mathrm{~cm}$ cement paste samples containing different fractions of fly ash, by frequency [7] 
Burnt bricks with a high volume ratio of fly ash are of high compressive strength, no cracking due to lime, poor water absorption, high resistance to excretion, no freezing and high resistance to frost melting.

\section{Carbon composites}

The two main types of carbon composites used for the purpose of electromagnetic shielding according to the study of carbon structures are carbon nanotubes and graphene [8]. Although several EM radiation particles such as carbon black, carbon fiber, or metallic fillers have been studied, carbon nanoparticles (CNT) have clearly shown better properties due to high ratio (length / height), higher strength and flexibility, and lower density, making them ideal as a filler [8].

There are two types of carbon nanotubes used, namely single-walled carbon nanotubes (SWCNT) and multi-walled carbon nanotubes (MWCNT). Comparing these two types, multi-walled carbon nanotubes (MWCNT) have higher protection efficiencies than single-walled carbon nanotubes (SWCNT). The most modern materials for protection against electromagnetic interference (EMI) are ultra-thin graphene composites with which a shielding efficiency of more than 20 $\mathrm{dB}$ can be achieved. This compound combines the excellent protective properties of graphene from EMI with the mechanical strength of the matrix material. Adding MWCNT to a concrete wall increases its shielding efficiency. The thicker the wall is reinforced with MWCNT, the higher its shielding efficiency. Figure 6 shows a comparison of the shielding efficiency of a concrete composite material M3 containing 3\% MWCMT of total weight for different thicknesses; the results are compared with the $S E$ provided by a $3 \mathrm{~cm}$ thick wall made of bare concrete.

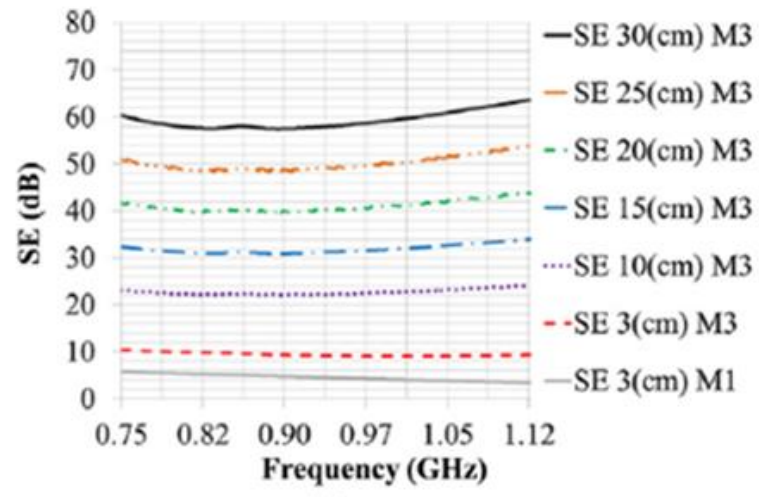

(a)

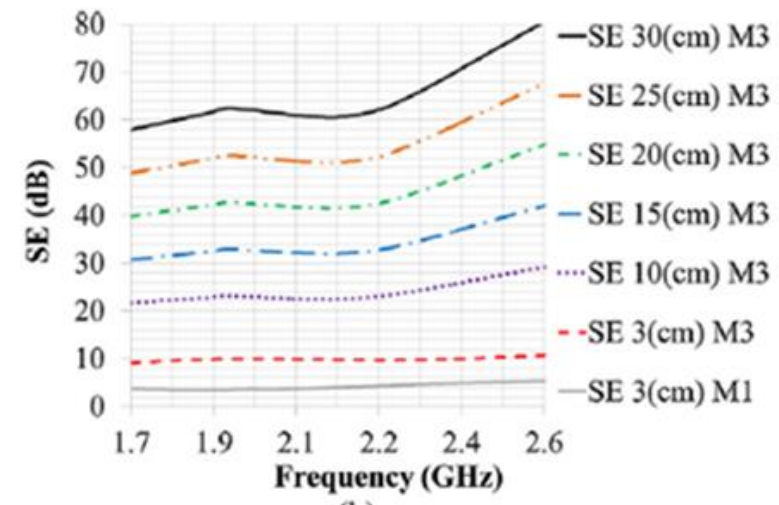

(b)

Fig. 6. Calculation of shielding efficiency of concrete composites reinforced with M3 material in the frequency band from 0.75 to $1.12 \mathrm{GHz}$ (a) and from 1.7 to $2.6 \mathrm{GHz}$ (b) [9]

Figure 7 shows the effect of clay on the electrical conductivity of SWCNT-filled epoxy composite. The conductivity of the epoxy composite is increased by the addition of SWCNT, and the addition of clay increases its dispersion. The addition of clay increases the viscosity of the composite mixture before hardening, which makes it difficult for carbon nanotubes to migrate and re-aggregate after ultrasound. Carbon nanotubes interact more strongly with clay than with epoxy.

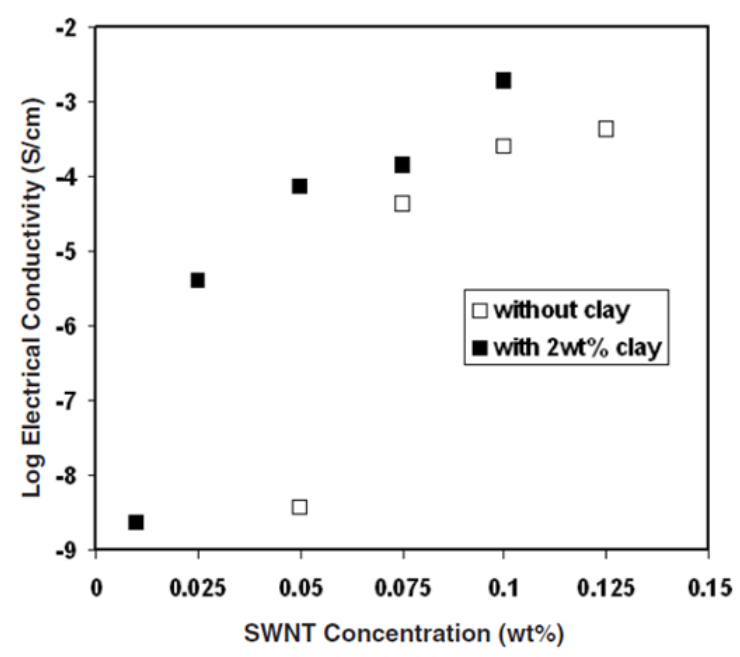

Fig. 7. Electrical conductivity as a function of SWCNT concentration for composites without clay and with the amount of clay $2 \%$ of the total mass of the composite [10] 


\section{Conclusion}

The main problem that prompted this study is the increasing use of electronic devices. The danger of EM radiation is encountered in everyday life and thus the danger of radiation is increasing. The solution to this problem is considered to be the innovation and use of materials that successfully absorb EM radiation. The ability of a particular material to absorb EM radiation is most often measured by the shielding efficiency. The total shielding efficiency of material against EM radiation penetration is obtained as the sum of the loss of absorption during wave transport in the medium and the loss of reflection which may include reflections when the waves are inside and outside the protective material.

As far as metallurgical slag is concerned, electric arc furnace slag (EAFS) has better EM radiation shielding efficiency, absorption coefficient and reflection coefficient than ladle furnace slag (LFS). The addition of waste tires also improves the EM radiation shielding efficiency, absorption coefficient and reflection of the material. Steel mini scale (ms) waste also serves to improve the shielding efficiency of material from EM radiation and absorption coefficient, while the reflection coefficient is approximately the same with and without steel mini scale (ms) waste. When comparing ceramic tiles and a brick wall reinforced with steel scrap, ceramic tiles have better protection effectiveness. Fly ash is one of the most well-known materials for improving the shielding efficiency of building materials from the EM radiation. Magnetic fly ash improves shielding efficiency the most while non-magnetic fly ash has lower protection efficiency compared to the original fly ash. Comparing carbon nanotubes, multilayer carbon nanotubes have better shielding efficiency of material protection against EM radiation than single-layer carbon nanotubes. The thicker the carbon-nanotubes-reinforced wall, the greater the shielding efficiency from EM radiation.

As for future research on this topic, the next step is to experimentally test the processed materials, and the ultimate goal is to innovate new materials that will have the ability to absorb EM radiation. New materials should be clay-based. The following research will be carried out as part of the project "Blocks of advanced materials that absorb EM radiation." These studies are expected to sufficiently investigate materials that enhance the absorption of EM radiation and how these materials will be successfully applied in practice.

\section{References}

[1] Sikalidis, C. (2011). Clay forms electromagnetic shield, Clay Technology Magazine, Aristotle University of Thessaloniki, Greece

[2] Lee, H. H., Yen, T. (2010). Upgrading on Cement-Mortar Properties for Electromagnetic Wave Shielding in Buildings, 2nd International Conference on Mechanical and Electronics Engineering (ICMEE)

[3] Bantsis, G., Sikalidis, C., Betsiou, M., Yioultsis, T., Bourliva, A. (2011). Ceramic building materials for electromagnetic interference shielding using metallurgical slags. Advances in Applied Ceramics, Vol. 110, No. 4, 233-237

[4] Bantsis, G., Mavridou, S., Sikalidis, C., Betsiou, M., Oikonomou, N., Youltsis, T., (2012). Comparison of low cost shielding-absorbing cement paste building materials in X-band frequency range using a variety of wastes, Science Direct, Ceramics International 38 3683-3692

[5] Bantsis, G., Sikalidis, C., Betsiou, M., Youltsis, T., Xenos, T., (2011). Electromagnetic absorption, reflection and interference shielding in $\mathrm{X}$-band frequency range of low cost ceramic building bricks and sandwich type ceramic tiles using mill scale waste as an admixture, Ceramics International 37 3535-3545, Science Direct

[6] Zhang, W., Zeng, J., Liu, L., Fang, Y. (2003). A novel property of styrene-butadiene-styrene/clay nanocomposites: radiation resistance, Material Chemistry, State University of New York at Stony Brook

[7] Zornoza, E., Catala, G., Jimenez, F., Andion, L. G., Garces, P., (2010). Electromagnetic interference shielding with Portland cement paste containing carbon materials and processed fly ash, Materiales de Construccion, Vol. 60, 300, 21-32, ISSN: 0465-2746, eISSN: 1988-3226, doi: 10.3989/mc.2010.51009

[8] Gonzalez, M., Mokry, G., de Nicolas, M., Baselga, J., Pozuelo, J., (2016). Carbon Nanotube Composites as Electromagnetic Shielding Materials in GHz Range, http://dx.doi.org/10.5772/62508

[9] Micheli, D., Vricella, A., Pastore, R., Delfini, A., Morles, R. B., Marchetti, M., Santoni, F., Bastianelli, L., Moglie, F., Mariani Primiani, V., Corinaldesi, V., Mazzoli, A., Donnini, J., (2017). Electromagnetic properties of carbon nanotube reinforced concrete, Construction and Building Materials, 131, 267-277

[10] Liu, L., Grunlan, J. C., (2007). Clay Assisted Dispersion of Carbon Nanotubes in Conductive Epoxy Nanocomposites, Advanced functional materials 17, 2343-2348, DOI: 10.1002/adfm.200600785

[11] Mann, H. S., Brar, G. S., Mann, K. S., Mudahar, G. S., (2016.). Experimental Investigation of Clay Fly Ash Bricks for Gamma-Ray Shielding, Nuclear Engineering and Technology 48, 1230-1236

[12] Wang, Z., Meng, X., Li, J., Du, X., Li, S., Jiang, Z., Tang, T., (2009). A Simple Method for Preparing Carbon Nanotubes/Clay Hybrids in Water, J. Phys. Chem. C, 113, 8058-8064

[13] Santangelo, S., Gorrasi, G., Di Lieto, R., De Pasquale, S., Patimo, G., Piperopoulos, P., Lanza, M., Faggio, G., Mauriello, F., Messina, G., Milone, C., (2011). Polylactide and carbon nanotubes/smectite-clay nanocomposites: Preparation, characterization, sorptive and electrical properties, Applied Clay Science 53, 188-194

[14] Bantsis, G., Betsiou, M., Bourliva, A., Youltsis, T., Sikalidis, C., (2012). Synthesis of porous iron oxide ceramics using Greek wooden templates and mill scale waste for EMI applications, Science Direct, Ceramics International $38,721-729$ 